\title{
Formation of layered fracture and outburst in stratums
}

\author{
X. B. Lu $\cdot$ X. H. Zhang $\cdot$ L. Lu
}

Received: 18 December 2013/Accepted: 17 October 2014/Published online: 1 November 2014

(C) Springer-Verlag Berlin Heidelberg 2014

\begin{abstract}
Layered fracture and outburst are two typical types of failures in a stratum that occur in many cases, such as liquefaction of sand, mining of coal, and dissociation of gas hydrate. The formation of both the failures is analyzed using the mathematical model based on the three-phase media. It is found that both failures occur once the pore pressure is high enough to cause the crashing and breakage of the skeleton. In other words, the formation conditions are related to the tensile strength of the stratum and the overburden. The fracture expands and shrinks along with the penetration and drainage of pore water and gas; outburst stops gradually as the energy dissipates in the stratum.
\end{abstract}

Keywords Layered fracture - Outburst · Gas hydrate . Liquefaction

\section{Introduction}

Layered fracture [also called water film (Fig. 1a)] and outburst in soil stratum occur in many cases, such as in the liquefaction of sand [also called soil boiling in liquefaction sand (Fig. 1b)] (Huang and Jiang 2010; Huang and Yu 2013; Lu and Cui 2004) in the mining of coal, and during the dissociation of gas hydrate. Layered fracture is a gap of water or gas in stratum and often spreads over a large area.

X. B. Lu $(\bowtie) \cdot$ X. H. Zhang

Institute of Mechanics, Chinese Academy of Sciences,

Beijing 100190, China

e-mail: xblu@imech.ac.cn

L. Lu

School of Science, China University of Mining and Technology, Beijing 100083, China
Outburst is strong eruption of soil-water/gas mixture from a hole or a vertical fracture in the stratum like volcanic explosion (Lu et al. 2006; Brennan and Madabhushi 2005).

Water film is first observed in liquefied sand containing an impermeable seam accompanying slope failures in earthquakes (Seed 1987), and its formation in stratified sand has been revealed by shake table tests (Kokusho 1999) and centrifuge tests (Fiegel and Kutter 1994). Moreover, it is found that the film develops just beneath the fine sand layer and generally forms the sliding surface of slope. Zheng et al. (2001) presented a model considering the erosion and re-deposition of fine grains in skeleton. They thought water films could be formed by the magnification of inhomogeneity through the transport of the fine grains (Zheng et al. 2001; Lu and Cui 2010). Malvick et al. (2008) discussed the development of water film using the concept of shear localization. But in many cases, the stratum bears no shearing.

Sand boiling results from liquefaction, which is an eruption of sand-water mixture out onto the ground surface, often occurring during/after earthquake. For example, sand boiling has been found in many sites during the 2008 Wenchuan earthquake and 1976 Tangshan earthquake in China. Huang and Yu (2013) classified the sand boiling formation into two categories: flat-cone sand volcanoes and liquefied sands erupting on the surface through existing cracks (Huang and Jiang 2010; Huang and Yu 2013). When the sand boils, it really liquefies and the hydraulic gradient is equal or larger than the over burden pressure (Wang 1997).

In the case of coal mining with complex geology and low strength of coal, the mines prone to fracture or burst depend on the tectonic and gas pressure. Experiments showed that the coal mine can be broken and gasified into outburst when the gas pressure is much higher than its 

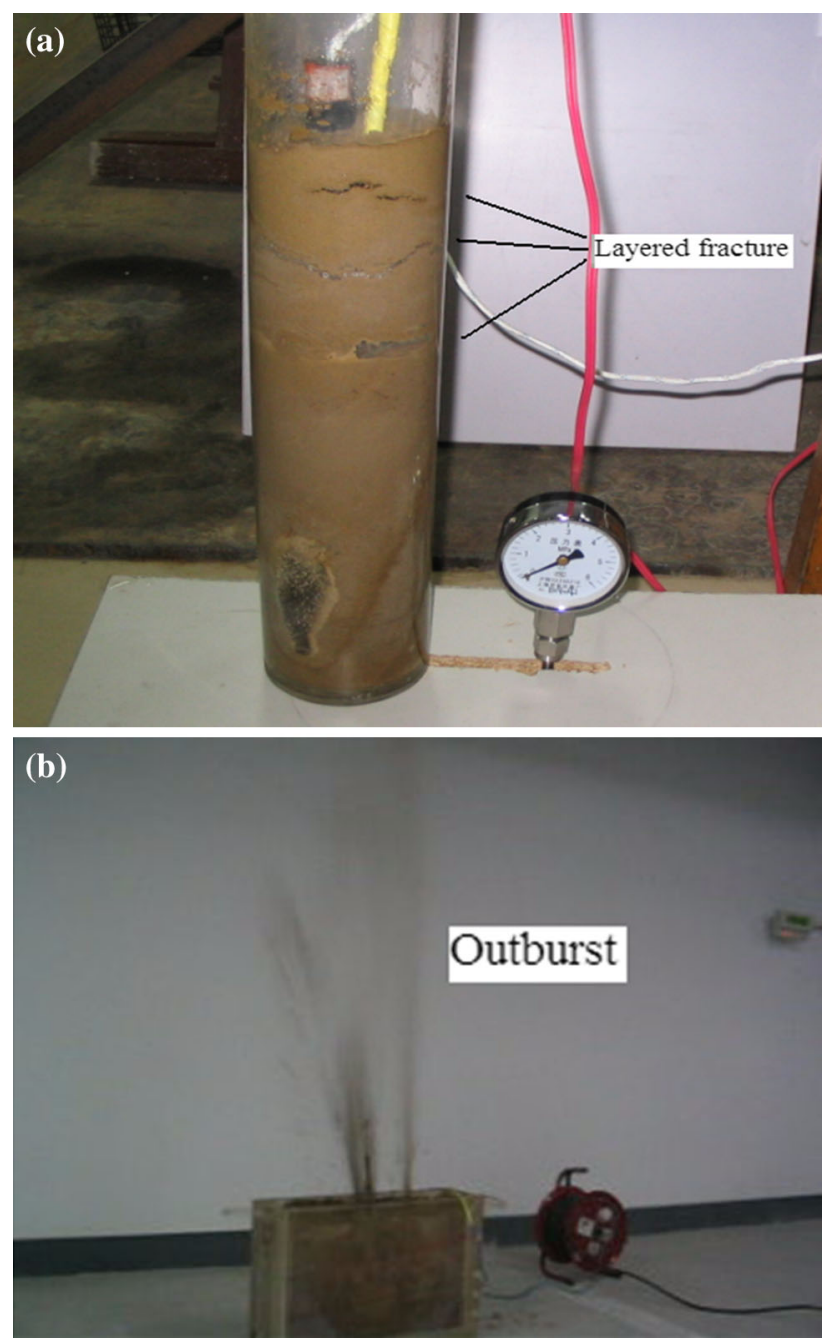

Fig. 1 Photos of layered fracture and outburst. a Water film in a liquefied sand layer and $\mathbf{b}$ outburst in a stratum after the dissociation of gas hydrate

tensile strength. The wave shape of pressure drop does not change with position. The product of width of coal fragment and the speed of damage front are linear with the difference of the pore pressure and the tensile strength. Only layered fracture occurs when the gas pressure is equal or a little higher than its tensile strength (Ding et al. 1989; Cheng and Ding 1987; Zheng et al. 1993). Numerical simulations investigated the effects of main factors such as sorption and coal strength on the formation of outburst (Sobczyk 2011; Xue et al. 2014). Yu et al. (1997) considered that the gas outburst during coal mining was discontinuous wave and presented a criterion. This criterion cannot be used in the formation of water film and outburst due to gas hydrate dissociation.

Dissociation of methane gas hydrate from a stratum usually produces gas and water. Generally, $1 \mathrm{~m}^{3}$ methane gas hydrate results in $164 \mathrm{~m}^{3} / \mathrm{m}^{3}$ methane gas and $0.27 \mathrm{~m}^{3}$ water at $1 \mathrm{~atm}$, and so causes a pore pressure as high as about $50 \mathrm{MPa}$ in the stratum if the gas cannot drain (Briaud and Chaouch 1997). The high pore pressure can cause the occurrence of layered fracture or outburst (Fig. 1c) (Zhang et al. 2011). The layered facture can trigger landslide of ocean floor and outburst can cause damage to anything in its path. They can also cause the escape of methane gas or hydrate from stratum. Released methane hydrate and/or bubbles from seabed may dissolve in water or rise to the ocean surface, and even drive a limnic-type eruption. This depends on the mixing of stratum. When the stratum is insignificant in mixture, methane hydrate may rise buoyantly through the water column either individually or collectively with other hydrate (Zhang 2003). There are gas, water and hydrate in pores after dissociation of gas hydrate; so the formation of layered fracture and outburst in this case is more complicated than in the cases of water film and sand boiling, and coal mining. Few theoretical analysis on the formation mechanism and process has been done up to now.

On the points of above, although these two types of failures have been found and studied for many years, most are in situ observation, in-room tests, and numerical simulations. Few theoretical analyses are conducted and the pore fluid is either water or gas, so the results cannot be used in conditions of pore fluids with two or more media. It is necessary to obtain theoretical explanations and criterion for the formation of layered fracture and outburst in extensive conditions.

This paper reports the theoretical study on the mechanism, and formation of layered fracture and outburst in a stratum. The formation of layered fracture and outburst is discussed in the second section and the third section, respectively. Experimental certification is processed in the fourth section. For the analysis conveniently, the discussions in this paper are based on one-dimensional model.

\section{Layered fracture}

Layered fracture happens when pore pressure reaches and exceeds the tensile strength of stratum and the over burden. The expansion of high-pressure pore fluid causes the overcap to form a gap; and the gap expansion in turn decreases the pore pressure down to below the pressure of the underneath zone and results in another gap when the pressure difference is large enough. A series of fractures may develop when such a process continues (Scotter 1986).

For the formation of layered fracture, the velocities of grains and pore fluids are neglectable, and the pore water and grains can be taken as incompressible. A one-dimensional model is presented as follows (Tan and Yu 1997; Zheng et al. 1993). The stratum is thought to be a threephase media consisting of gas, water, and grains so that the 
model can be used in more extensive conditions than the previous models. Thus, the mathematic model includes mass equilibrium equations and momentum equilibrium equations of these three phases, respectively.

The mass equilibrium equations of gas, water, and grains are as follows

$\frac{\partial \varepsilon_{i} \rho_{i}}{\partial t}+\frac{\partial \varepsilon_{i} \rho_{i} u_{i}}{\partial x}=0$,

in which $\varepsilon_{i}, \rho_{i}, u_{i}$ indicate the concentrations, densities, and velocities of gas, water, and grains corresponding to $i=$ $1,2,3$ respectively, $x$ is the coordinate, and $t$ is time.

The momentum equilibrium equations of gas and water are adopted as:

$\varepsilon_{j} \rho_{j} \frac{\mathrm{d} u_{j}}{\mathrm{~d} x}+\varepsilon_{j} \frac{\mathrm{d} p_{j}}{\mathrm{~d} x}=-H_{j}-\varepsilon_{j} \rho_{j} g$,

in which $j=1,2$ indicate gas and water, respectively, $p$ is the pore pressure, $H_{j}(j=1,2)$ are the resistances between water and skeleton and between gas and skeleton, respectively, $H_{j}=\frac{\varepsilon_{j}^{3}}{k_{j}}\left(u_{j}-u_{\mathrm{s}}\right), k_{j}$ are the physical permeabilities of gas and water, respectively, corresponding to $j=1,2$.

The momentum equilibrium equation of the grains is as follows:

$$
\begin{gathered}
\varepsilon_{\mathrm{s}} \rho_{\mathrm{s}} \frac{\mathrm{d} u_{\mathrm{s}}}{\mathrm{d} x}+\frac{\mathrm{d} \varepsilon_{\mathrm{s}} \sigma}{\mathrm{d} x}+p_{\mathrm{g}} \frac{\mathrm{d} \varepsilon_{\mathrm{g}}}{\mathrm{d} x}+p_{\mathrm{w}} \frac{\mathrm{d} \varepsilon_{\mathrm{w}}}{\mathrm{d} x} \\
=H_{\mathrm{w}}+H_{\mathrm{g}}+\frac{\tau l}{A}-\varepsilon_{\mathrm{s}} \rho_{\mathrm{s}} g,
\end{gathered}
$$

in which $\rho_{\mathrm{s}}$ is the density of the skeleton, $u_{\mathrm{s}}$ the velocity of the skeleton, $\varepsilon_{\mathrm{S}}$ the concentration of the skeleton, $\varepsilon_{\mathrm{g}}$ the concentration of the pore gas, $\varepsilon_{\mathrm{w}}$ the concentration of the pore water, $\sigma$ the total stress, $p_{\mathrm{g}}$ the pore gas pressure, $p_{\mathrm{w}}$ the pore water pressure, $H_{\mathrm{w}}$ the resistance between pore water and skeleton, $H_{\mathrm{g}}$ the resistance between pore gas and skeleton, $\tau l / A$ the side friction in unit length, $A$ the cross section area, and $l$ the side length.

The total momentum equilibrium equation of the stratum can be obtained by summing up Eqs. (2) and (3):

$$
\begin{aligned}
& \varepsilon_{\mathrm{s}} \frac{\partial \sigma}{\partial x}+\varepsilon_{\mathrm{w}} \frac{\partial p_{\mathrm{w}}}{\partial x}+\varepsilon_{\mathrm{g}} \frac{\partial p_{\mathrm{g}}}{\partial x}-\varepsilon_{\mathrm{m}} \rho_{\mathrm{m}} g-\varepsilon_{\mathrm{w}} \rho_{\mathrm{w}} g-\varepsilon_{\mathrm{g}} \rho_{\mathrm{g}} g \\
& \quad=\varepsilon_{\mathrm{m}} \rho_{\mathrm{m}} \frac{\partial u_{\mathrm{m}}}{\partial t}+\varepsilon_{\mathrm{w}} \rho_{\mathrm{w}} \frac{\partial u_{\mathrm{w}}}{\partial t}+\varepsilon_{\mathrm{g}} \rho_{\mathrm{g}} \frac{\partial u_{\mathrm{g}}}{\partial t}+p_{\mathrm{a}}+\frac{\tau l}{A},
\end{aligned}
$$

where the inertial terms, $\partial u_{\mathrm{m}} / \partial t, \partial u_{\mathrm{w}} / \partial t$, and $\partial u_{\mathrm{g}} / \partial t$, can be ignored when the skeleton is stiff and the seepage is small. Then the layered fracture forms under the following condition as integrating Eq. (4) in vertical direction:

$$
\begin{aligned}
p= & \varepsilon_{\mathrm{g}} p_{\mathrm{g}}+\varepsilon_{\mathrm{w}} p_{\mathrm{w}}>\sigma_{\mathrm{t}}+\varepsilon_{\mathrm{m}} \rho_{\mathrm{m}} g L+\varepsilon_{\mathrm{w}} \rho_{\mathrm{w}} g L+\varepsilon_{\mathrm{g}} \rho_{\mathrm{g}} g L \\
& +\frac{\tau l}{A} L+p_{\mathrm{a}},
\end{aligned}
$$

in which $\sigma_{\mathrm{t}}$ is the tensile strength of the stratum, $p_{\mathrm{a}}$ the atmospheric pressure, $p=\left(\varepsilon_{\mathrm{w}} p_{\mathrm{w}}+\varepsilon_{\mathrm{g}} p_{\mathrm{g}}\right)$ the pore pressure,

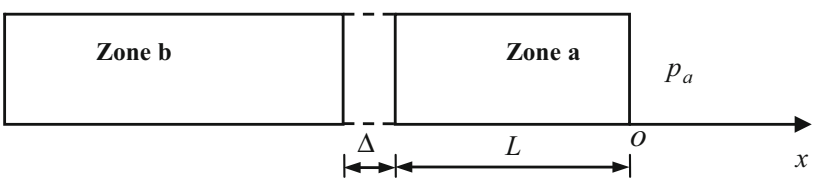

Fig. 2 Sketch of layered fracture

and $L$ the length of the moving part (Zone a in Fig. 2). The gravity terms occur only when the failure happens in the vertical direction. Actually, the earth gravity of the stratum can be neglected since it is much smaller than the pore pressure in most cases. Then the critical condition reduces to

$p \geq p_{\mathrm{a}}+\frac{\tau l}{A} L+\sigma_{\mathrm{t}}$.

For zone a in Fig. 2, the following equations are adopted:

$p-p_{\mathrm{a}}-\frac{\tau l}{A} L=\rho_{\mathrm{s}}\left(1-\varepsilon_{0}\right) L \cdot \ddot{\Delta}$,

i.c., $t=0, \Delta=\Delta_{0}, \dot{\Delta}=0$,

in which $\varepsilon_{0}$ is the initial porosity, $\Delta$ is the width of the fracture, and $\Delta_{0}$ is the initial width of the fracture which is assumed as a smallness. The left-hand side of Eq. (7) is the driving force that pore pressure overcomes the resistances and the right is the movement.

With the movement of the skeleton, the fracture expands and the pore pressure inside of it decreases to be lower than that outside of it; then the pore fluids penetrate into it from outside to cause the pore pressure inside of it to increase.

The seepage of pore gas is assumed to satisfy the Darcy law and the gas is perfect. The seepage equation and mass equilibrium equation of pore gas satisfy the following:

$\left\{\begin{array}{c}-\frac{\partial p_{\text {ig }}}{\partial x}=\frac{\varepsilon_{0} \mu_{\mathrm{g}}}{k_{\mathrm{g}}} u_{\mathrm{ig}} \\ \frac{\partial \rho_{\mathrm{ig}}}{\partial t}+\frac{\partial \rho_{\mathrm{ig}} u_{\mathrm{ig}}}{\partial x}=0 \\ p_{\mathrm{ig}}=\rho_{\mathrm{ig}} a^{2}\end{array}\right.$.

Similarly, the seepage equation and mass equilibrium equation of pore water satisfy the following:

$\left\{\begin{array}{c}-\frac{\partial p_{\text {iw }}}{\partial x}=\frac{\varepsilon_{0} \mu_{\mathrm{w}}}{k_{\mathrm{w}}} u_{\mathrm{iw}} \\ \frac{\partial \rho_{\mathrm{w}}}{\partial t}+\frac{\partial \rho_{\mathrm{w}} u_{\mathrm{ww}}}{\partial x}=0\end{array}\right.$,

in which $i$ indicates the Zone a and Zone b in Fig. 2 corresponding to $i=1,2$, respectively, $\mu$ is the viscosity of pore fluid, $k$ is the permeability, subscripts $g, w$ indicate gas and water, respectively, and $a$ is the sonic speed. 
Assume the initial pore gas (or water) pressure is $\mathrm{p}_{0 \mathrm{~g}}$ (or $\left.\mathrm{p}_{0 \mathrm{w}}\right)$ throughout the stratum. In Zone a, the right end is opened to the atmosphere, so the pressure is $1 \mathrm{~atm}$ both for pore gas and water. At the left end, the pressures of pore gas and water are equal to that inside the fracture, respectively. Thus, the initial and boundary conditions for pore gas and water are as follows:

i.c., $\quad u_{\mathrm{ag}}=0, p_{\mathrm{ag}}=p_{0 \mathrm{~g}}$,

$b . c, x=-L, p_{\mathrm{ag}}=p_{\mathrm{g}} ; x=0, p_{\mathrm{ag}}=p_{\mathrm{a}}$,

i.c., $\quad u_{\mathrm{aw}}=0, p_{\mathrm{aw}}=p_{0 \mathrm{w}}$,

$b . c, x=-L, p_{\mathrm{aw}}=p_{\mathrm{w}} ; x=0, p_{\mathrm{aw}}=p_{\mathrm{a}}$.

Similarly, the initial and boundary conditions for Zone $b$ are

i.c., $\quad u_{\mathrm{bg}}=0, p_{\mathrm{bg}}=p_{0 \mathrm{~g}}$,

$b . c, x=-\infty, p_{\mathrm{bg}}=p_{0 \mathrm{~g}} ; x=-L, p_{\mathrm{bg}}=p_{\mathrm{g}}$,

i.c., $\quad u_{\mathrm{bI}}=0, p_{\mathrm{bw}}=p_{0 \mathrm{w}}$,

b.c $, x=-\infty, p_{\mathrm{bw}}=p_{0 \mathrm{w}} ; x=-L, p_{\mathrm{bw}}=p_{\mathrm{w}}$.

The pore gas pressure and the water pressure are connected by the capillary force $p_{\mathrm{c}}$ :

$p_{\mathrm{g}}-p_{\mathrm{w}}=p_{\mathrm{c}}$.

The flow rates $\mathrm{Q}_{\mathrm{a}}$ and $\mathrm{Q}_{\mathrm{b}}$ from Zone $\mathrm{a}$ and Zone $\mathrm{b}$ into the fracture are as follows:

$Q_{\mathrm{a}}=\varepsilon_{0} A\left(\rho_{\mathrm{aw}} u_{\mathrm{aw}}+\rho_{\mathrm{ag}} u_{\mathrm{ag}}\right)$,

$Q_{\mathrm{b}}=\varepsilon_{0} A\left(\rho_{\mathrm{bw}} u_{\mathrm{bw}}+\rho_{\mathrm{bg}} u_{\mathrm{bg}}\right)$.

The mass of the pore fluid in the fracture consisted of that flowing from Zones $\mathrm{a}$ and $\mathrm{b}$ and the initial mass. The width of the fracture is mainly determined by the volume occupied by the gas because the density of water is very larger than that of the gas in the case of gas hydrate dissociation or mining of coal. It is determined by pore water in the case of liquefaction. So the pore fluid mass and the fracture width can be expressed as follows:

$m=m_{\mathrm{g}}+m_{\mathrm{w}}=m_{0 \mathrm{~g}}+m_{0 \mathrm{w}}+\int_{0}^{t} Q_{\mathrm{a}} \mathrm{d} t+\int_{0}^{t} Q_{\mathrm{b}} \mathrm{d} t$,

$\Delta=\Delta_{\mathrm{g}}+\Delta_{\mathrm{w}}=\frac{m}{A \cdot \rho_{\mathrm{g}}}$,

in which $m_{0 w}$ and $m_{0 g}$ are the initial masses of pore water and pore gas in the fracture $\Delta_{0}$, respectively. The increase of pore pressure and the expansion of the fracture width are induced mainly by the gas during/after gas hydrate dissociation.

The pore gas pressure in the fracture can be obtained using the isothermal relation:

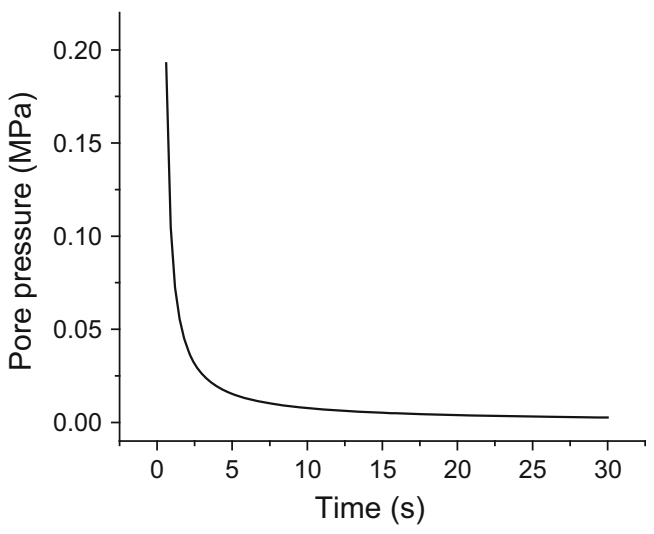

Fig. 3 The development of pore pressure in the fracture

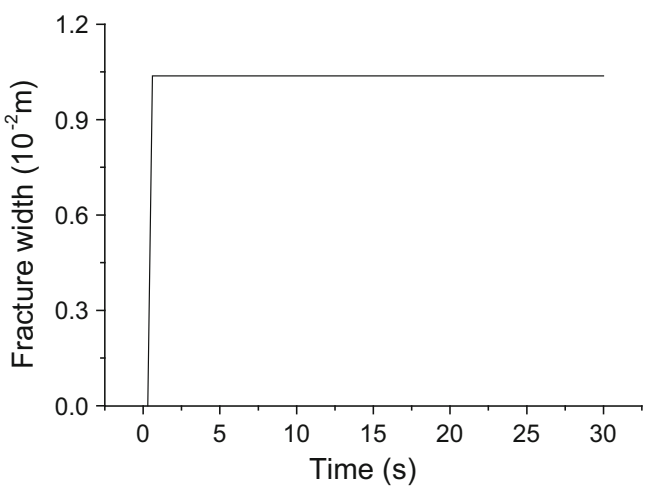

Fig. 4 Development of the fracture width

$p_{\mathrm{g}}=\rho_{\mathrm{g}} \cdot c^{2}=\frac{m_{\mathrm{g}}}{A \cdot \Delta_{\mathrm{g}}} c^{2}$,

in which $a$ is the local sonic speed.

Finite difference method is used for analysis of Eqs. (9)(24), adopting the parameters: $\rho_{\mathrm{s}}=1,600 \mathrm{~kg} / \mathrm{m}^{3}$, $\rho_{\mathrm{w}}=1,000 \mathrm{~kg} / \mathrm{m}^{3}, \varepsilon_{0}=0.4, \tau L / A=1.9 \mathrm{MPa} / \mathrm{m}, \sigma_{\mathrm{t}}=0$, $p_{\mathrm{a}}=0.1 \mathrm{MPa}, \quad \mu=1.5 \times 10^{-5}$ Pa.s, and $k=1.0 \times$ $10^{-12} \mathrm{~m}^{2}$ (Zhang et al. 2011).

The pore pressure in the fracture deceases very fast in the first $5 \mathrm{~s}$ and then continues to decrease slowly (Fig. 3), in agreement with the experimental results shown in Fig. 9 in some extend. The fracture width increases fast at first and then approaches a constant (Fig. 4). The pore pressure decreases very fast and cannot drive the moving of Zone a with the expansion of the fracture; so the fracture width is limited.

\section{Outburst}

When the pore pressure is much higher than the tensile strength of the skeleton and the over burden, and the 


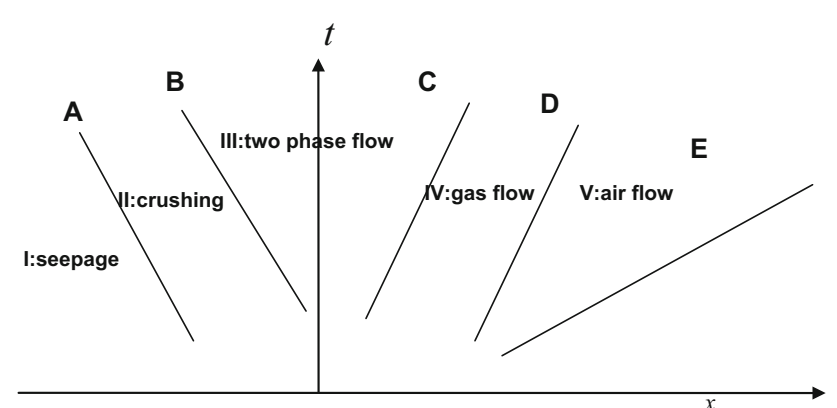

Fig. 5 Sketch of the problem

stratum is weak in a local zone like a vertical fracture or an abandoned well, outburst occurs often. According to experiments, at first, the free gas and water (released from gas hydrate or coal or liquefied soils) are obstructed in certain regions to accumulate (Zhang et al. 2011; Zhang 2010; Lu et al. 2004). At this stage, seepage and the deformation of the skeleton are very small. Gradually, the pore pressure increases and so the seepage velocity increases and some of the skeleton begin to be crushed. Thus, the movement of the skeleton becomes obvious. With more and more of the skeleton being crushed, the fast flow of the mixture containing grains, gas, and water forms, i.e., outburst. Following this fast mixture flow, the gas and water penetrate the rest zone slowly. So the stratum during outburst can be divided into five zones from its depth to the surface (Fig. 5):

Zone I: deep in the stratum, seepage is dominated and movement of skeleton is neglectable.

Zone II: crushing initiates from Line A and two-phase flow begins to form, ending at Line $\mathrm{B}$ when the velocities of the skeleton and the pore fluid are equal.

Zone III: two-phase flow forms completely (outburst).

Zone IV: there are pure gas and water flow.

Zone V: there is only constant flow of gas or water following the outburst.

Outburst processes steadily in experiments, i.e., with a constant velocity w (Cheng and Ding 1987). With coordinate transformation $\xi=x+w t$, the Eqs. (1)-(5) become

$s_{\mathrm{g}} \rho_{\mathrm{g}}\left(w+u_{\mathrm{g}}\right)=\varepsilon_{0 \mathrm{~g}} \rho_{0 \mathrm{~g}} w$,

$s_{\mathrm{w}} \rho_{\mathrm{w}}\left(w+u_{\mathrm{w}}\right)=\varepsilon_{0 \mathrm{w}} \rho_{0 \mathrm{w}} w$,

$(1-\varepsilon) \rho_{\mathrm{s}}\left(w+u_{\mathrm{s}}\right)=\left(1-\varepsilon_{0}\right) \rho_{\mathrm{s}} w$,

$s_{\mathrm{g}} \rho_{\mathrm{g}}\left(w+u_{\mathrm{g}}\right) \frac{\mathrm{d} u_{\mathrm{g}}}{\mathrm{d} \xi}+s_{\mathrm{g}} \frac{\mathrm{d} p_{\mathrm{g}}}{\mathrm{d} \xi}=-H_{\mathrm{g}}$,

$s_{\mathrm{w}} \rho_{\mathrm{w}}\left(w+u_{\mathrm{w}}\right) \frac{\mathrm{d} u_{\mathrm{w}}}{\mathrm{d} \xi}+s_{\mathrm{w}} \frac{\mathrm{d} p_{\mathrm{w}}}{\mathrm{d} \xi}=-H_{\mathrm{w}}$,

$$
\begin{aligned}
& (1-\varepsilon) \rho_{\mathrm{s}}\left(w+u_{\mathrm{s}}\right) \frac{\mathrm{d} u_{\mathrm{s}}}{\mathrm{d} \xi}+\frac{\mathrm{d}[(1-\varepsilon) \sigma]}{\mathrm{d} \xi}+p \frac{\mathrm{d} \varepsilon}{\mathrm{d} \xi}=H-\frac{\tau l}{A} \\
& s_{\mathrm{g}} \rho_{\mathrm{g}}\left(w+u_{\mathrm{g}}\right) \frac{\mathrm{d} u_{\mathrm{g}}}{\mathrm{d} \xi}+s_{\mathrm{w}} \rho_{\mathrm{w}}\left(w+u_{\mathrm{w}}\right) \frac{\mathrm{d} u_{\mathrm{w}}}{\mathrm{d} \xi} \\
& \quad+(1-\varepsilon) \rho_{\mathrm{s}}\left(w+u_{\mathrm{s}}\right) \frac{\mathrm{d} u_{\mathrm{s}}}{\mathrm{d} \xi}+\frac{\mathrm{d}[(1-\varepsilon) \sigma]}{d \xi}+\frac{\mathrm{d} p_{\mathrm{g}} s_{\mathrm{g}}}{\mathrm{d} \xi}+\frac{\mathrm{d} p_{\mathrm{w}} \varepsilon_{\mathrm{w}}}{\mathrm{d} \xi} \\
& \quad=-\frac{\tau l}{A},
\end{aligned}
$$

in which $\varepsilon=s_{\mathrm{g}}+s_{\mathrm{w}}$, and $\varepsilon_{0}=s_{\mathrm{g} 0}+s_{\mathrm{w} 0}$. For simplicity, the following assumptions are adopted: the pore fluid is a mixture with density $\rho=s_{\mathrm{g}} \rho_{\mathrm{g}}+s_{\mathrm{w}} \rho_{\mathrm{w}}$, the pore pressure is $p=s_{\mathrm{g}} p_{\mathrm{g}}+s_{\mathrm{w}} p_{\mathrm{w}}$, and $s_{\mathrm{g}}, s_{\mathrm{w}}$ are the proportions of gas and water vapor, respectively.

During the seepage stage (Zone I), the following equations are satisfied: $\varepsilon=\varepsilon_{0}, u_{\mathrm{s}}=0, p=c^{2} \rho, H=\frac{\varepsilon_{0}^{2} \mu}{k}$, and $k$ is the physical permeability of the pore fluid.The pore pressure at the end connected with Zone I and Zone II equals to $\mathrm{p}_{1}$, and much inside Zone I equals to the initial pore pressure. Thus, the boundary conditions are as follows:

$\xi=0, p=p_{1}, \xi=-\infty, p \rightarrow p_{0}$.

Equations (25) and (26) yield

$\frac{p}{p_{0}}=\frac{1}{1+\frac{u}{w}}$.

Equations (29) and (30) together with $w<<a$ and $p \approx$ $p_{0}$ yield

$\frac{\mathrm{d} \sigma^{\prime}}{\mathrm{d} \xi}+\frac{\mathrm{d} p}{\mathrm{~d} \xi}=-\frac{\tau l}{A}$,

in which $\sigma^{\prime}=\left(1-\varepsilon_{0}\right)(\sigma-p)$ is the effective stress. In this case, $\tau$ can be thought as a ultimate side friction stress $\tau_{\mathrm{M}} \cdot \tau=\tau_{\mathrm{M}}$ at $\xi \rightarrow 0$ and $\tau=0$ at $\xi<<0$.

So

$\sigma^{\prime}=-\frac{\tau_{\mathrm{M}} l}{A} \xi+p_{1}-p$.

The condition should be satisfied at the point T (Fig. 6):

$\sigma^{\prime}=-\sigma_{\mathrm{t}}, \frac{\mathrm{d} \sigma^{\prime}}{\mathrm{d} \xi}=0$.

So

$p_{\mathrm{T}}=\frac{1}{1+\xi}$,

And

$\frac{\tau_{M} l}{A p_{0}} \xi_{T}=s-p_{T}+p_{1}$, 


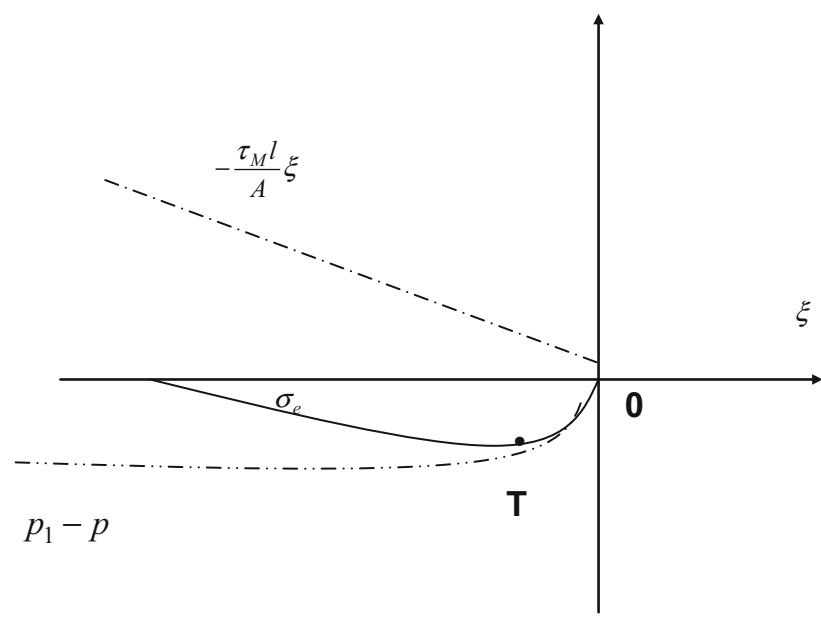

Fig. 6 Sketch of the distribution of the effective stress

in which $p_{\mathrm{T}}=\frac{p_{\mathrm{T}}}{p_{0}}, s=\frac{\sigma_{\mathrm{t}}}{p_{0}}$, and $\eta=\frac{\tau_{\mathrm{M}} l k}{A \varepsilon_{0} \mu w}$.

$\frac{1-p_{1}}{1-p_{\mathrm{T}}}-\ln \frac{1-p_{1}}{1-p_{\mathrm{T}}}=1+\frac{s}{\eta}$.

Comparing the definition with Eqs. (33) and (37), $\eta$ can be obtained as

$\eta=\frac{u_{\mathrm{T}}}{w}$.

The crushing course is very complex from Line A to the end of Line B. Only the total momentum Eq. (31) is considered here. Meanwhile, the shear stress is assumed to be zero.

Adding Eqs. (30) and (31) together, and then integrating by neglecting the smallness, $\mathrm{p}_{2}$ can be expressed as

$p_{2}=p_{1}-\left(1-\varepsilon_{0}\right) \rho_{\mathrm{s}} w u_{2}$.

From Eq. (27), $\varepsilon_{2}$ can be determined

$\varepsilon_{2}=\frac{\frac{u_{2}}{w}+\varepsilon_{0}}{\frac{u_{2}}{w}+1}$.

From Eqs. (25) and (26), $\rho_{2}$ can be obtained as

$\rho_{2}=\frac{\varepsilon_{0} \rho_{0}}{\frac{u_{2}}{w}+\varepsilon_{0}}$.

The equation of energy conservation is as follows

$$
\begin{aligned}
& \frac{\varepsilon_{0}}{1-\varepsilon_{0}} \frac{\rho_{0}}{\rho} \frac{\gamma}{\gamma-1}\left(\frac{p_{2}}{\rho_{2}}-a^{2}\right)+\frac{1}{\rho_{\mathrm{s}}}\left(p_{2}-p_{1}\right) \\
& +\frac{1}{2}\left(w+u_{2}\right)^{2}-\frac{1}{2} w^{2},
\end{aligned}
$$

and

$p_{2}=p_{\mathrm{a}}+\rho_{\mathrm{a}} c u_{2}$

in which $p_{\mathrm{a}}$ is the atmosphere pressure and $\rho_{\mathrm{a}}$ is the density at 1 atm.
Generally, there exists a minimum gas pressure $p_{\mathrm{cr}}$ for steady outburst. By Eqs. (37), (38), (41) and (45), $p_{\text {cr }}$ can be obtained as follows

$$
\begin{aligned}
p_{\mathrm{cr}}= & p_{\mathrm{a}}+\sigma_{\mathrm{t}}+\left[\left(1-\varepsilon_{0}\right) \rho_{\mathrm{s}} w_{\mathrm{cr}}+\rho_{\mathrm{a}} c\right] u_{2 \mathrm{cr}}-\frac{\tau_{\mathrm{M}} l}{A} \xi_{\mathrm{Tcr}} \\
& +\frac{u_{\mathrm{T}}}{w_{\mathrm{cr}}} p_{\mathrm{T}} .
\end{aligned}
$$

According to Eqs. (40) and (46), the relations between $p_{\text {cr }}$ and $u_{\mathrm{T}}$ and between $\varepsilon_{0}$ can be determined, which are shown in Figs. 7 and 8 by adopting the parameters in literature (Zhang et al., 2011). It can be seen that $p_{\text {cr }}$ increases with $u_{\mathrm{T}}$ fast at first and then slowly, but decreases with $\varepsilon_{0}$ linearly. $u_{2}$ can be obtained as $0.12 \mathrm{~m} / \mathrm{s}$. Here the following parameters are adopted in the computation: $p_{\mathrm{a}}=0.1 \mathrm{MPa}, \sigma_{\mathrm{t}}=0 \mathrm{MPa}, \tau_{\mathrm{M}}=0.08 \mathrm{MPa}, \varepsilon_{0}=0.4$, $\rho_{\mathrm{s}}=1,600 \mathrm{~kg} / \mathrm{m}^{3}, \quad w_{\mathrm{cr}}=0.2 \mathrm{~m} / \mathrm{s}, \quad u_{\mathrm{t}}=0.01 \mathrm{~m} / \mathrm{s}$, $\rho_{\mathrm{a}}=2.9 \mathrm{~kg} / \mathrm{m}^{3}, \quad c=350 \mathrm{~m} / \mathrm{s}, \quad \xi=0.01 \mathrm{~m}, \quad$ and $1 /$ $A=0.05$.

Figure 7 Relation between $p_{\mathrm{cr}}$ and $u_{\mathrm{T}}$ Fig. 8 Relation between $p_{\mathrm{cr}}$ and $\varepsilon_{0}$

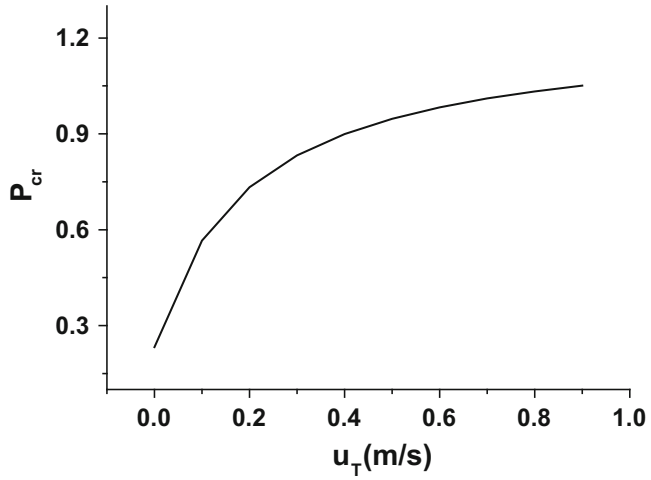

Fig. 7 Relation between $p_{\text {cr }}$ and $u_{\mathrm{T}}$

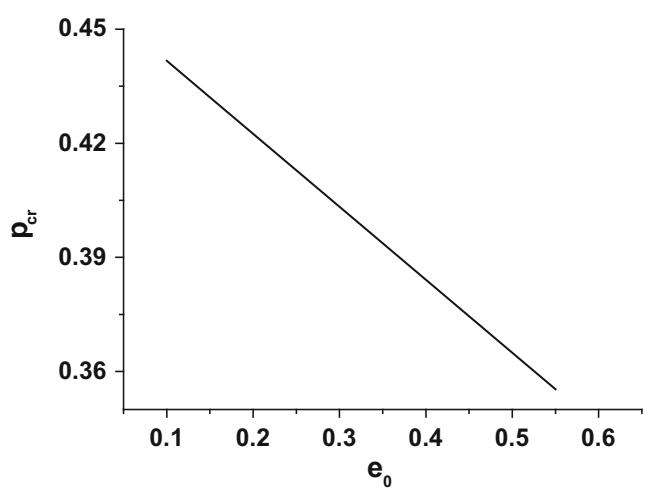

Fig. 8 Relation between $p_{\text {cr }}$ and $\varepsilon_{0}$ 


\section{Experimental certification}

One-dimensional experiments were conducted to verify the critical conditions for layered fracture and outburst. Pore pressure was induced by GH dissociation. The dissociation rate was controlled by the temperature of heat source. The pore pressure and the side friction were controlled by the drained conditions which were determined by the thickness of over layer. In this way, experiment can be designed to observe the formation of layered fracture and outburst. Here two types of over-cap, $5 \mathrm{~cm}$ silty sand layer and $5 \mathrm{~cm}$ hydrate deposit (HD), were set up, respectively, to investigate the effects of the drainage condition. Two experiments were carried out with over-cap of $5 \mathrm{~cm}$ silty sand layer (drained condition) and one experiment was adopted over-cap of $5 \mathrm{~cm} \mathrm{HD}$ (undrained condition).

Experiments were carried out in an organic glass cylinder with inner size of diameter $\times$ height $=10 \times 30 \mathrm{~cm}$. An immersion heater of $400 \mathrm{~W}$ with length $\times$ diameter $=12 \times 1 \mathrm{~cm}$ was vertically placed $10 \mathrm{~cm}$ below the over-cap (Zhang et al. 2011) to control the temperature and provide heat for GH dissociation.

During preparing samples, silty sand with a density of $1,600 \mathrm{~kg} / \mathrm{m}^{3}$ was first compacted to make skeleton and then saturated by Tetrahydrofuran (THF) liquor with a mass fraction of $19 \%$.

According to experimental observations, the pore pressure near the heater rose to about $0.22 \mathrm{MPa}$ in the first 2 min once the immersion heater was switched on. This value keeps as almost a constant with the expansion of dissociation zone in about 10-20 min changing with the power of heater. When the dissociation zone expands to the critical scale and accordingly the pore pressure is accumulated to be larger than the stratum's strength and the side friction, the fracture initiates and expands. Then the pore pressure decreased very fast in the first $10 \mathrm{~s}$ and then continues to decrease slowly (Fig. 9). In Fig. 9, only the later part that the pore pressure decreases from the peak is

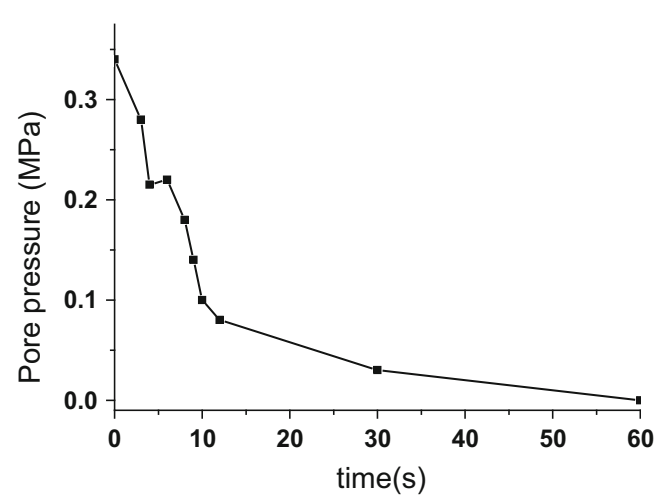

Fig. 9 Pressure-temperature evolution curve shown for comparing with the theoretical results (shown in Fig. 3) conveniently and clearly. By the state equation of perfect gas $P=N R T / V,\left(R=8.31 \mathrm{~Pa}^{3} \mathrm{~m}^{3} \mathrm{~mol}^{-1} \mathrm{~K}^{-1}, N\right.$ is the Mole number, $T$ temperature, $V$ volume, and $P$ pressure), NRT can be thought as an constant in the experiment, the fracture develops to the same order as the pore space in the dissociation zone in a short time. So pore pressure decreases very fast once it initiates. The decrease rate is related with the permeability in the dissociation zone. That is why the pore pressure drops rapidly once the fracture initiates. Layered fractures were observed in the first two experiments using a partially drainage over layer and outburst is observed in the third experiment adopted an impermeable over layer. The gas pressures in the first two experiments were less than that in the other experiments because the pore pressure can dissipate partially. The maximum pore pressure prior to layered fracture failure is $0.22 \mathrm{MPa}$. The maximum gas pressure rose to $0.3 \mathrm{MPa}$ before the outburst. The side friction was measured as $\tau l L / A=0.108 \mathrm{MPa}, \quad l / A=10$, and $L=0.03 \mathrm{~m}$. After hydrate dissociation, both the tensile strength and the side stress $\sigma_{\mathrm{s}}$ of the hydrate-bearing stratum equal zero and the Poisson's ratio is about 0.15 .

According to Eqs. (10) and (46), the theoretical critical pore pressure for layered fracture and outburst are 0.208 and $0.29 \mathrm{MPa}$, respectively, which are close to the experimental data.

\section{Discussion and conclusions}

The formation of layered fracture and outburst in a stratum is discussed based on a one-dimensional mathematical model. The critical conditions and the evolution corresponding to these two failure modes are obtained and compared with experimental results.

It is shown that once the pore pressure is equal or larger than the tensile strength, layered fracture or outburst may happen in a liquefied or gasified stratum.

Water and gas trapped by the over layer with relatively low permeability in a heterogeneous stratum may cause the pore pressure to accumulate. Once the pore pressure rises to exceed the overburden and the side friction, and the tensile strength of the stratum, and meanwhile there is no downward drainage through the over layer, the relative movement between the upward pore fluid flow and the settlement of grains at the interface must be equal to the velocity of settlement at the upper liquefied or gasified soil surface, the over layer will be pushed forward to form a fracture or even crushed.

Formation of a fracture may lead to a sudden unloading of the pore pressure. Accordingly, an unloading wave will transmit into the stratum and leads to tensile stresses whose 
magnitude depends on the pressure difference between the pressures inside and outside the fracture. If the pressure difference is large enough, more fractures can occur. In the limit case, the distance between every two neighbor fractures becomes very short, then outburst occurs.

The critical condition of layered fracture is mainly related to the tensile strength of the stratum and the side friction. The critical condition of outburst is mainly related to the velocity of the skeleton and the maximum pore pressure except for the tensile strength of the stratum and the side friction.

Acknowledgments This paper is supported by The National Natural Science Foundation of China (No. 11272314; No. 51239010; No. 11102209).

\section{References}

Brennan AJ, Madabhushi SPG (2005) Liquefaction and drainage in stratified soil. ASCE J Geotech Geoenviron Eng 131(7):876-885

Briaud JL, Chaouch A (1997) Hydrate melting in soil around hot conductor. ASCE $\mathrm{J}$ Geotech and Geoenviron Engrg 123(7):645-653

Cheng CM, Ding YS (1987) A preliminary study of gas burst. In: Proceeding of Int Symp Mining Tech Science, China Coal Industry Publishing House, Beijing, pp 366-377

Ding XL, Yu SB, Ding YS, Kou SQ, Tan QM, Zheng ZM (1989) Mechanism for the continuous damage of coal under the gas seepage. Sci China A 6:601-607

Fiegel GL, Kutter BL (1994) Liquefaction mechanism for layered sands. ASCE J Geotech Eng 120(4):737-755

Huang Y, Jiang XM (2010) Field-observed phenomena of seismic liquefaction and subsidence during the 2008 Wenchuan earthquake in China. Nat Hazard 54:839-850

Huang Y, Yu M (2013) Review of soil liquefaction characteristics during major earthquakes of the twenty-first century. Nat Hazards 65:2375-2384

Kokusho T (1999) Water film in liquefied sand and its effect on lateral spread. ASCE J Geotech Geoenviron Eng 125(10):817-826

Lu XB, Cui P (2004) The liquefaction and displacement of highly saturated sand under water pressure oscillation. Ocean Eng $31: 795-811$
Lu XB, Cui P (2010) On the study of water film in saturated soils. Int J Sediment Res 25:221-232

Lu XB, Tan QM, Wang SY (2004) The advances of liquefaction research on saturated soils. Adv Mech 34(1):87-96

Lu XB, Wu YR, Zheng ZM (2006) Formation mechanism of cracks in saturated sand. Acta Mech Sin 22(4):377-383

Malvick EJ, Kutter BL, Boulanger RW (2008) Postshaking shear strain localization in a centrifuge model of a satuarted sand slope. ASCE J Geotech Geoenviron Eng 134(2):164-174

Scotter RF (1986) Solidification and consolidation of a liquefied sand column. Soils Found 26(4):23-31

Seed HB (1987) Design problems in sand liquefaction. ASCE J Geotech Eng 113(8):827-845

Sobczyk J (2011) The influence of sorption processes on gas stresses leading to the coal and gas outburst in the laboratory conditions. Fuel 90:1018-1023

Tan QM, Yu SB (1997) Fracture of coal containing pressurized gas by sudden relieving. J China Coal Soc 22(5):514-518

Wang WS (1997) Dynamic strength and liquefaction properties of soil. China Electric Power Press, Beijing

Xue S, Yuan L, Wang YC, Xie J (2014) Numerical analysis of the major parameters affecting the initiation of outburst of coal and gas. Rock Mech Rock Eng 47:1505-1510

Yu SB, Cheng CM, Tan QM, Ding YS (1997) Damage of porous dedia containing pressurized gas by unloading and the maximum damage principle for critical outburst. Acta Mech Sin 29(6):641-646 (in Chinese)

Zhang YX (2003) Methane escape from gas hydrate systems in marine environment, and methane-driven oceanic eruptions. Geophys Res Lett 30(7):51-1-51-4

Zhang XH (2010) Study on the softening and damage of sediment induced by thermal dissociation of gas hydrate. Thesis for $\mathrm{Ph}$. D Institute of Mechanics, Chinese Academy of Sciences, Beijing

Zhang XH, Lu XB, Li QP (2011) Formation of layered fracture and outburst during gas hydrate dissociation. J Petrol Eng Sci $76: 212-216$

Zheng ZM, Chen L, Ding YS (1993) Steady advance of damage front caused by gas outburst under one dimensional condition. Sci China A 23(4):723-730

Zheng ZM, Tan QM, Peng FJ (2001) On the mechanism of the formation of horizontal cracks in a vertical column of saturated sand. Acta Mech Sin 17(1):1-9 